\title{
Mobile Phone Based Falling Detection Sensor and Computer-Aided Algorithm for Elderly People
}

\author{
Jong-Ha Lee , Hee-Jun Park , and Seon-Chil Kim \\ Department of Biomedical Engineering, School of Medicine, Keimyung University, Daegu, South Korea
}

\begin{abstract}
Falls are dangerous for the elderly population; therefore many fall detection systems have been developed. However, previous methods are bulky for elderly people or only use a single sensor to isolate falls from daily living activities, which makes a fall difficult to distinguish. In this paper, we present a cost-effective and easy-to-use portable fall-detection sensor and algorithm. Specifically, to detect human falls, we used a three-axis accelerator and a three-axis gyroscope in a mobile phone. We used the Fourier descriptor-based frequency analysis method to classify both normal and falling status. From the experimental results, the proposed method detects falling status with $96.14 \%$ accuracy.
\end{abstract}

\section{Introduction}

Falling, which means losing one's balance or tripping over something, occurs in all age groups, but especially in the elderly. In Asia, senior citizens over the age of 65 experience over half of the body injuries caused by falling [1]. In spite of extensive preventive efforts, falls remain the leading cause of accidental deaths among the elderly. Research estimates that over a third of the people aged over 65 suffer from a fall each year, and the number of falls increases progressively with age in both genders and in all racial and ethnic groups [2]. Because of vision and other problems, the falls typically occur in certain environmental conditions. Falls cause serious damage, including fractures, brain damage, and anxiety toward exercise. Quickly dealing with a fall injury such as a broken bone will reduce the time the bone fragment remains in the body. Moreover, some fractured bone regions never completely heal if they do not receive proper care within four hours [3]. If a person falls and bumps his or her head, he or she can black out, and any resulting brain damage should be cared for in a short amount of time to reduce side effects such as paralysis. Reducing the time until treatment can also help prevent a second injury and pain. For rapid treatment, the most important challenge is fall detection, which simultaneously provides communication of the fall. Therefore, fall detection is critical. A mobile phone with a three-axis accelerometer and gyroscope is a device that can easily carried by humans. In this paper, a mobile phone is used to classify human falls from normal walking motion. The three-axis accelerometer is used to measure three direction activity data. A gyroscope sensor is used to detect angular momentum; it is also useful in determining the user's rotation when he or she falls. To verify the accuracy of the proposed method, the system and algorithm were validated with a real human data set.

\section{Materials and Method}

\subsection{Data acquisition}

Subjects either walked or fell down while holding a mobile phone in their hand. A three-axis accelerometer was used to detect users' activity such as walking, falling, sitting, etc. When a person falls, one's body rotates on the body's center. A gyroscope that measures angular momentum was used to detect this rotation. The sampling frequency of each data was $20 \mathrm{~Hz}$. The device used for the data acquisition was the Galaxy Note 1 (Samsung Electronics, Seoul, South Korea). The total data set of walking and falling was 212 and 202, respectively. The mean age of the subjects was 23.2; the age range was from 21 to 24 .

\subsection{Data analysis}

For analysis and synthesis of the plane closed curves, the Fourier descriptor (FD) is used. The normalized Fourier transformed coefficients are called the Fourier descriptors [4]. The discrete Fourier transformation of the signature function is given by the following equation:

$$
a_{n}=\frac{1}{N} \sum_{t=0}^{N-1} r(t) \exp \left(\frac{-j 2 \pi n t}{N}\right), n=0,1, \cdots, N-1
$$


The data was produced using a one-point fast Fourier transformation. The FD was extracted from the $\mathrm{x}$ accelerometer, $\mathrm{y}$-accelerometer, $\mathrm{z}$-accelerometer, $\mathrm{x}$ gyroscope, y-gyroscope, and z-gyroscope signals: a total of 96 features were considered. Figure 1 shows the $\mathrm{x}$-axis accelerometer signal while walking (up) and falling (down) and Figure 2 presents the $\mathrm{x}$-axis gyroscope signal while walking (up) and falling (down). From the results, we clearly see the difference between the accelerometer signal and the gyroscope signal while walking and falling in the time domain.
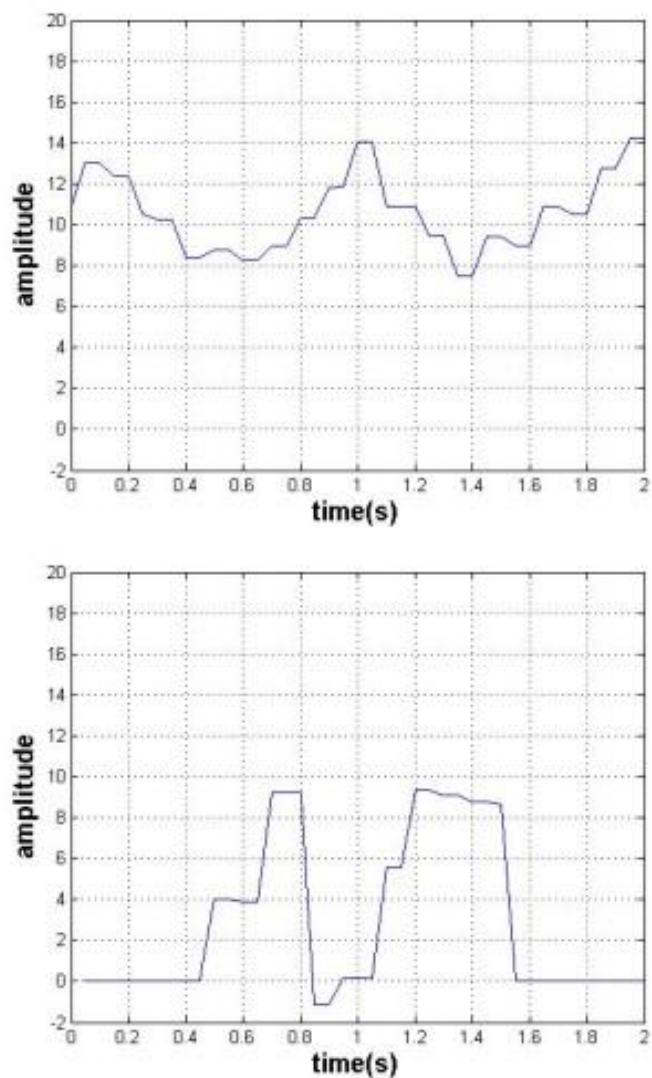

Figure 1. X-axis accelerometer signal while walking (up) and falling (down).

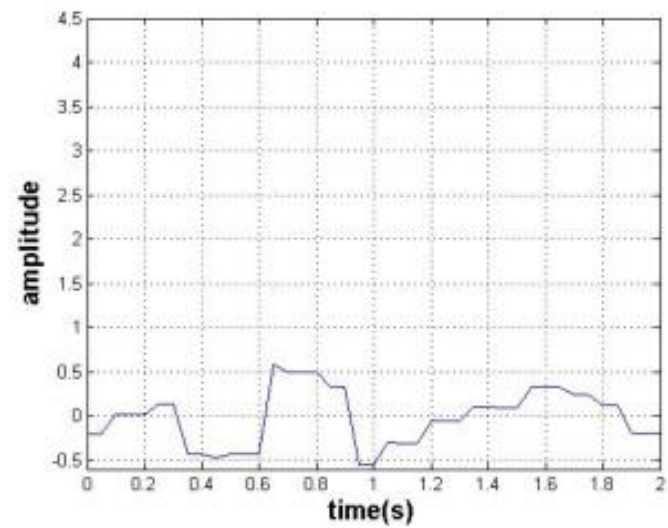

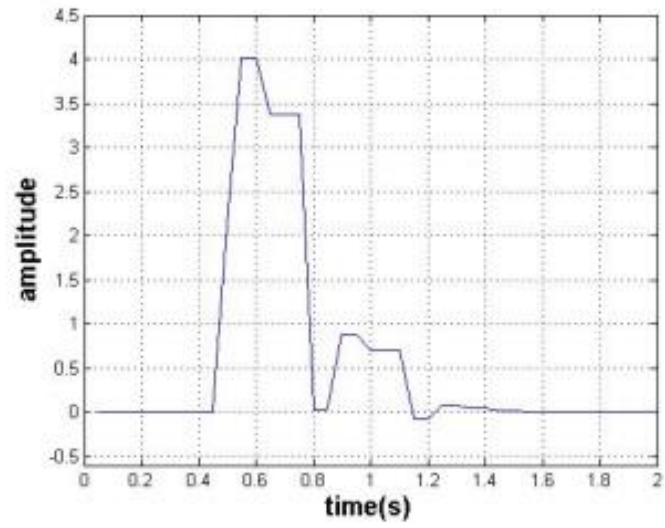

Figure 2. $\mathrm{X}$-axis gyroscope signal while walking (up) and falling (down).

\subsection{Fourier Descriptor}

For analysis and synthesis of the plane-closed curves, the Fourier descriptor (FD) is used. FD is obtained by applying Fourier transform on a shape signature derived from shape boundary coordinates in Section III-A and IIIB.

The normalized Fourier transformed coefficients are called the Fourier descriptors. The discrete Fourier transform of signature function is given by

$$
a_{n}=\frac{1}{N} \sum_{t=0}^{N=1} r(t) \exp \left(\frac{-j 2 \pi n t}{N}\right), n=0,1, \ldots, N=1
$$

Since the CDF and CSCF are invariant to translation and rotation, the Fourier coefficients have to be further to make them scale invariant and start point independent shape descriptors. The relation between Fourier coefficients of original shape and transformed through scaling and change of start point is given by

$$
a_{n}=\exp (j n \varphi) \cdot s \cdot a_{n}^{o}
$$

where $\varphi$ is the angles incurred by the change of start point and and $s$ is the scale factor. Then normalized Fourier coefficients $b_{n}$ of the transformed shape is obtained by

$$
b_{n}=\frac{a_{n}}{a_{1}}=\frac{a_{n}^{o}}{a_{1}^{o}} \exp [j(n-1) \varphi]=b_{n}^{o} \exp [j(n-1) \varphi]
$$

where $b_{n}^{o}$ is the normalized Fourier coefficients of the original shape. From Eq. (4), it is clear that, if we ignore phase of the coefficients, then magnitudes $\left|b_{n}\right|$ and $\left|b_{n}^{o}\right|$ are the same. In other words, $\left|b_{n}\right|$ is invariant to translation, rotation, scaling and change of start point. The values of $\left|b_{n}\right|$ are the shape features that we used for the breast tumor classification. For the improvement in the classification of ultrasound images, we have combined both Fourier descriptors from CDF and CSCF through staked vector approach (SVA) technique, where both Fourier descriptors are concatenated to form a long feature vector. 


\subsection{Support vector machine (SVM) classifier}

The support vector machine (SVM) is a widely used method for classification and regression tasks. For a twoclass problem with training samples $\left\{x_{i}, y_{i}\right\}, \mathrm{i}=1,2, \cdots, \mathrm{N}$ in d-dimensional feature space, $x_{i} \in R^{d}$, with associated targets $y_{i} \in\{-1,1\}$, the discriminate function of the separating hyperplane for linearly separable classes is given as follows:

$$
f(x)=w \cdot \Phi(x)+b
$$

where $\mathrm{w} \in R^{d}$ is the vector normal to the hyperplane and $\mathrm{b} \in \mathrm{R}$ is the bias. In addition, $x_{i}$ is the featured vectors extracted from the gyroscope and accelerometer sensors and $y_{i}$ is the falling and normal status.

The SVM fits a hyperplane to the training samples of two classes in the feature space by minimizing the cost function, which consists of two criteria: margin maximization and error minimization [5]. SVM used real number vector. In this paper, a two-category walk or fall can be showed as $(1,0)$ and $(0,1)$.

\subsection{Evaluation model}

The model's accuracy is estimated using a crossvalidation method. The data is divided into $\mathrm{K}$ to $\mathrm{K}$-fold cross-validation [6]. The subsample left validation data. The other (K-1) data use training data. Validation data is tested using the classifier trained on the remaining training data. In addition, $\mathrm{K}$ is repeated, changing the subsample. Each instance of the whole training set is predicted once so the cross-validation accuracy is the percentage of data, which are correctly classified [7]. After that, the results take an average. $Y_{i}$ is the real response values and $X_{p}$ is the vector covariates.

$$
\frac{\sum_{i}\left(Y_{i}-a-b_{1} X_{1 i}-\cdots-b_{p} X_{p i}\right)^{2}}{n}, \mathrm{n}=\mathrm{K}, \mathrm{i}=1,2, \cdots \mathrm{n}, \mathrm{p}=1,2, \cdots \mathrm{n}
$$

TP and TN are the correctly classified occurrences of either walking or falling. On other hand, walking and falling are incorrectly classified as FP and FN. An evaluation was completed using a tenfold cross-validation.

\section{Experimental Results}

To calculate the classification accuracy, the K-fold crossvalidation method was used and $K$ was set as 10 . The performance indices were evaluated by five performance indices, including classification accuracy $(\mathrm{TP}+\mathrm{TN}) /(\mathrm{TP}+\mathrm{TN}+\mathrm{FP}+\mathrm{FN})$, sensitivity $(\mathrm{TP} /[\mathrm{TP}+\mathrm{FN}])$, specificity $(\mathrm{TN} /[\mathrm{TN}+\mathrm{FP}])$, positive predictive value $(\mathrm{TP} /[\mathrm{TP}+\mathrm{FP}])$, and negative predictive value $(\mathrm{TN} /[\mathrm{TN}+\mathrm{FN}])$, where $\mathrm{TP}$ is the number of true positive findings correctly classified as positive; $\mathrm{TN}=$ true negative; $\mathrm{FP}=$ false positive; and $\mathrm{FN}=$ false negative [8]. From the results, we noticed that the proposed method detects 384 data (falling or walking) out of 414 original data (falling or walking). The statistical analysis shows that the resulting accuracy was $96.14 \%$, sensitivity was $100 \%$, specificity was $92.08 \%$, positive predictive value was $92.98 \%$, and negative predictive value was $100 \%$.

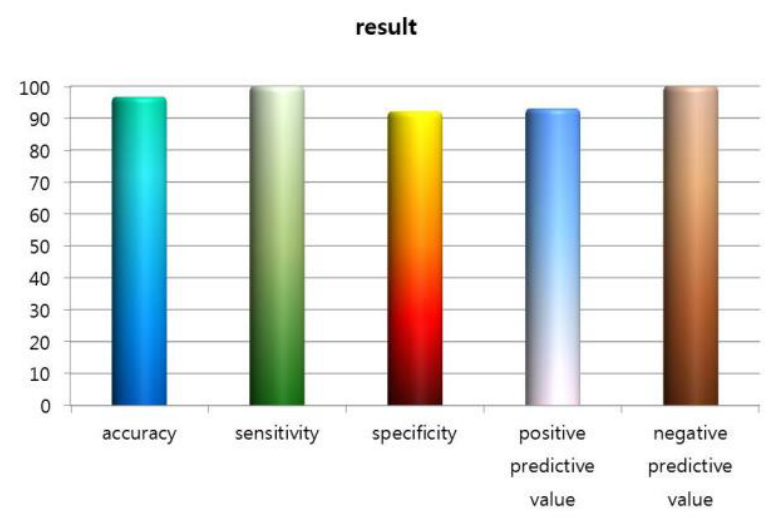

Figure 3. Statistical analysis results for proposed method verification.

\section{Discussion}

In this paper, we assumed that the elderly person always carries the proposed platform due to the fact that the ordinal mobile phone motion sensors are what detect the falling status. The current platform can be interrupted with events such as an incoming call, or if the motion sensors are occupied with other applications, the motiondetecting algorithm might be hold. Further, we assumed that the small and portable sensor attached to the elderly person will be carried by the same elderly person. Finally, the deliberate fall of young people is quite different from the fall of elderly people, and their walking patterns might also be quite different. In future work, the clinical trials of real data sets from both old and young people will be obtained and analyzed to optimize the fall detection algorithm for ordinary people.

\section{Conclusion}

In this paper, we propose a mobile phone-based falling detection sensor and algorithm. We used a three-axis accelerator and three-axis gyroscope to determine the direction and rotation of the subjects' bodies. The support vector machine classifier was used to analyze the sensor's signal. From the experimental results, the proposed method detects falling status with $96.14 \%$ accuracy, which indicates that human activity can be classified using a mobile phone. These detection methods can warn others of a user's fall.

\section{Acknowledgements}

The research partly was supported by the Ministry of Science, ICT and Future Planning Through the Development for IT $\cdot \mathrm{SW}$ industrial convergence original technology (ID: R0101-15-0147) and Basic Science Research Program through the National Research Foundation of Korea (NRF) funded by the Ministry of 
Education (2014R1A1A2056420) and supported by the Technological Innovation R\&D Program (S2173477) funded by the Small and Medium Business Administration (SMBA, Korea).

\section{References}

1. M.C. Hornbrook, V.J. Stevens, D.J. Wingfield, J.F. Hollis, M.R. Green lick, and M.G. Ory, Preventing falls among community-dwelling older persons: Results from a randomized trial, The Gerontologist 34, 16-23 (1994).

2. Y. Li, G. Chen, Y. Shge, Y. Zhu, and Z. Cheng, Accelerometer-based fall detection sensor system for the elderly, IEEE CCIS (2012).

3. T.S. Sorensen, H. Colding, C. Schroeder, and V.T. Rosdahl, The penetration of cefazolin, erythromycin and methicillin into human bone tissue 49, 549-553 (1978).

4. C.T. Zhan and R.Z. Roskies, Fourier descriptors for plane closed curves, IEEE Trans Computer 21, 269-281 (1972).

5. J.-H. Lee and Y. K. Seong, Fourier-based shape feature extraction technique for computer-aided b-mode ultrasound diagnosis of breast tumor, $34^{\text {th }}$ Annual Internal IEEE EMBS Conference (2012).

6. R.M. Simon, J. Subramanian, M.-C. Li, and S. Menezes, Using cross-validation to evaluate predictive accuracy of survival risk classifiers based on high-dimensional data, Oxford Journal 12, 203-214 (2011),

7. C.-W. Hsu, C.C. Chang, and C.-J. Lin, A practical guide to support vector classification, https://www.cs.sfu.ca /people/Faculty/teaching/726/spring11/svmguide.pdf (2003).

8. R.M. Rangan, Biomedical image analysis, CRC Press (2005). 